LU TP 11-06

MCnet/11/03

January 2011

\title{
Multiparton Interactions with an $x$-dependent Proton Size
}

\author{
R. Corke 11 and T. Sjöstrand? \\ Theoretical High Energy Physics, \\ Department of Astronomy and Theoretical Physics, \\ Lund University, \\ Sölvegatan 14A, \\ S-223 62 Lund, Sweden
}

\begin{abstract}
Theoretical arguments, supported by other indirect evidence, suggest that the wave function of high- $x$ partons should be narrower than that of low$x$ ones. In this article, we present a modification to the variable impact parameter framework of PYTHIA 8 to model this effect. In particular, a Gaussian hadronic matter profile is introduced, with a width dependent on the $x$ value of the constituent being probed. Results are compared against the default single- and double-Gaussian profiles, as well as an intermediate overlap function.
\end{abstract}

${ }^{1}$ richard.corke@thep.lu.se

${ }^{2}$ torbjorn@thep.lu.se 


\section{Introduction}

Multiparton interactions (MPI) are an unavoidable consequence of colliding hadrons at high energies. The theoretical description of soft MPI poses particular challenges, due to the limited understanding of non-perturbative QCD. Unfortunately, it is exactly this physics which is vital for describing minimum-bias (MB) and underlying events (UE) at hadron colliders, such as the LHC. To this end, phenomenological models are often introduced, to provide the best description possible.

The original MPI model, introduced in earlier versions of PYTHIA, extends the perturbative picture down to very low $p_{\perp}$ scales, such that one can view all events as containing one or more interactions [1-3]. These low- $p_{\perp}$ interactions fill the role of cut Pomerons [4,5], stretching colour fields longitudinally across an event, which later fragment. Of course, these colour fields can also stretch to higher- $p_{\perp}$ partons, giving a smooth transition to (mini)jets, and a unified picture of $\mathrm{MB}$ and UE physics. This model has been updated in recent times, and forms a part of the interleaved parton shower and MPI framework of PүтніA 8 [6].

Many other models for the structure of hadronic events have been formulated, that are all based on some kind of multiple interactions framework, be it in the form of soft or (semi)hard interactions, or a mixture thereof [10]. A few implementations are formulated with a view to be used also for hard-scale physics within and beyond the Standard Model [11], such as Herwig [12 17] and Sherpa [18, 19]. Others put more emphasis on the soft physics aspects, including the relations between elastic, diffractive and non-diffractive topologies, using concepts such as Dual Topological Unitarization [20] and Reggeon Field Theory [21]. Examples thereof include Phojet [22, 23], Dpmjet [24], Epos [25], Sibyll [26], and QGSJET [27].

In all of these programs the proton is handled as an extended object. That way an eikonal description [28] can be used, wherein the probability for an event to be produced is largest for head-on collisions and decreases for increasing impact parameter. The standard assumption for most of these scenarios is that the partons are distributed inside the protons according to a Gaussian, with the same radius for all parton species and momenta. There is no specific reason for this ansatz, but it makes for simple algebra in going from a three-dimensional spherical ansatz to a two-dimensional impact-parameter plane, and for convoluting these distributions for the two colliding hadrons. Other shapes have been used, e.g. the electromagnetic form factor in HERWIG, and some PYTHIA alternatives to be described later. A collision-energy-dependent radius is often used, and sometimes two different radii for soft and hard interactions, but these possibilities still offer fairly little flexibility. The one notable exception we are aware of is DIPSY [29 31], see further below.

The key objective of the current article will thus be to study the consequences if one of the conventional constraints is relaxed, namely that high- and low-momentum partons have the same impact-parameter profile. This should actually be considered as the expected behaviour, rather than an exotic variant, as follows.

The size of the proton is finite, owing to confinement, but exactly how it should be defined is ambiguous. Low-energy measurements give a root-mean-squared (RMS) charge radius $\approx 0.88 \mathrm{fm}[32$. Combined with the mass gap of QCD - the lightest free state being the pion - this leads to a finite proton-proton strong-interaction cross section $\sigma_{\mathrm{pp}}$ (while the electromagnetic one is infinite, the photon being massless). 
This cross section can vary as a function of energy, but its growth is limited by the Froissart-Martin bound [33, 34]. The intuitive idea underlying this bound is that the pion Yukawa potential fall-off $g e^{-m_{\pi} r} / r$ sets the maximum impact parameter $b_{\max }$ of interactions to be roughly where $|g| \exp \left(-m_{\pi} b_{\max }\right)=1$, i.e. $\sigma \simeq \pi b_{\max }^{2} \simeq\left(\pi / m_{\pi}^{2}\right) \ln ^{2}|g|$. Since it can be shown that $|g|$ can increase at most like a power of the collision energy under general conditions which should hold for QCD, it follows that $\sigma \propto \ln ^{2} s$ provides an upper bound. The numerical prefactor to the bound [35] is far from saturated at current energies, however, and work to improve on it is ongoing [36, 37].

The experimental observation of an increasing total cross section is reinforced by studies of the differential elastic cross section [38, 39], from which it is concluded that the proton gets "blacker, edgier, larger" with increasing energy [40,41].

By Gribov theory, the high- $s$ behaviour can be related to a low- $x$ one, with the size of the proton growing proportionally to $\ln (1 / x)$. Qualitatively (but not quantitatively, see below) this can be understood as a transverse random walk [42] in a BFKL [43, 44] evolution, where a few initial high- $x$ partons fairly close to the center of the proton emit a cascade of partons towards lower $x$ scales, and in the process these partons diffuse to be spread over a larger area. A more formal definition can be obtained by the Balitsky-JIMWLK evolution equations for hadronic amplitudes [45], which also can be described by the Color Glass Condensate formalism [46].

Mueller's dipole cascade model [47,48] offers a formulation of the BFKL evolution in transverse coordinate space, and so gives direct access to information on the spread of partons at different $x$ scales. The DIPSY generator provides a complete implementation, where effects of energy-momentum conservation, saturation, gluon recombination and the running of $\alpha_{\mathrm{s}}$ are consistently taken into account. One important message that comes out of the numerical studies is that the Froissart-Martin bound is violated asymptotically in the evolution equations, unless confinement is also built into the gluon propagator, in which case the $\ln ^{2} s$ behaviour is nicely obtained [49.

The DiPsy generator can also be used to study a number of further issues, such as diffraction [50] and elliptic flow [51]. So far it has not been used for comparisons with MB event properties at hadron colliders, however, and is not well suited for UE studies.

Generalized parton distributions offer an alternative approach to explore the transverse size of the proton [52, and to understand some MPI phenomenology [53. Again an $x$ dependence is obtained, where the proton radius vanishes in the limit $x \rightarrow 1$ (in part a natural consequence of a center-of-gravity definition of the origin).

In the current article we will not attempt to trace the evolution of cascades in $x$. Rather we will assume that the impact-parameter distribution of partons at any $x$ can be described by a simple Gaussian, $\exp \left(-b^{2} / a^{2}(x)\right)$, with a width that grows like $a(x)=a_{0}\left(1+a_{1} \ln (1 / x)\right)$. The coefficients $a_{0}$ and $a_{1}$ are tuned to the parameterised shape of $\sigma_{\mathrm{pp}}(s)$ in the following. The potential overlap between two protons will be described only in terms of their size at their respective $x$ values. In principle one should also include a third scale, related to the transverse distance the exchanged propagator particle, normally a gluon, could travel. This distance should be made dependent on the $p_{\perp}$ scale of the interaction. For simplicity we will not consider this further complication here, and only take the propagator distance into account by allowing a finite effective radius also for $x \rightarrow 1$. For minimum-bias studies, the results will be rather insensitive to the behaviour at large $x$ since, at high energies, the bulk of MPI occurs at small $x$. This choice will play more of a 
role in the underlying event of hard processes, but is not studied further.

In Section 2, some relevant aspects of the existing MPI model are given, before the modified impact parameter framework is introduced. Some results are shown in Section 3 , both in comparison to other matter profiles and to data, before a summary and outlook is given in Section 4 .

\section{Multiparton interaction framework}

The starting point is the hadronic perturbative cross section

$$
\frac{\mathrm{d} \sigma}{\mathrm{d} p_{\perp}^{2}}=\sum_{i, j} \iint \mathrm{d} x_{1} \mathrm{~d} x_{2} f_{i}\left(x_{1}, Q^{2}\right) f_{j}\left(x_{2}, Q^{2}\right) \frac{\mathrm{d} \hat{\sigma}}{\mathrm{d} p_{\perp}^{2}},
$$

where $\mathrm{d} \hat{\sigma} / \mathrm{d} p_{\perp}^{2}$ gives the partonic QCD $2 \rightarrow 2$ cross section, and $f_{i}$ and $f_{j}$ the PDF factors of the two incoming hadrons. In the modelling of soft MPI activity, there are two key observations that can be made. First, the QCD $2 \rightarrow 2$ cross section contains a $1 / p_{\perp}^{4}$ divergence in the $p_{\perp} \rightarrow 0$ limit. Second, the total integrated cross section down to some low- $p_{\perp}$ limit

$$
\sigma_{\text {hard }}\left(p_{\perp \text { min }}\right)=\int_{p_{\perp \text { min }}^{2}}^{s / 4} \frac{\mathrm{d} \sigma}{\mathrm{d} p_{\perp}^{2}} \mathrm{~d} p_{\perp}^{2},
$$

becomes comparable to the total cross section for $p_{\perp \text { min }} \approx 2-5 \mathrm{GeV}$ at current collider energies.

The original MPI model addresses these issues as follows [1]. It is observed that $\sigma_{\text {hard }}$ gives the hadron-hadron cross section and not the parton-parton one. If, in one hadron collision, many parton-parton interactions are possible, then $\langle n\rangle\left(p_{\perp \text { min }}\right)=\sigma_{\text {hard }}\left(p_{\perp \text { min }}\right) / \sigma_{\text {tot }}$ gives the average number of parton-parton scatterings above $p_{\perp \text { min }}$ per event. In dealing with non-diffractive inelastic events only, as in this article, the cross section for hard interactions, $\sigma_{\text {hard }}\left(p_{\perp \text { min }}\right)$, must be distributed among the $\sigma_{\mathrm{ND}}$ events, such that $\langle n\rangle\left(p_{\perp \text { min }}\right)=\sigma_{\text {hard }}\left(p_{\perp \text { min }}\right) / \sigma_{\mathrm{ND}}$.

This is still not a solution to the divergence of the cross section in the $p_{\perp} \rightarrow 0$ limit. The average $\hat{s}$ of scatterings decreases slower with $p_{\perp \text { min }}$ than the number of interactions increases, which would lead to an infinite amount of scattered partonic energy. One part of the solution is the need to include longitudinal correlations, including energy and momentum conservation effects. In the most recent iterations of the model, this is handled using a model dependent PDF rescaling procedure [6].

This effect alone is too weak, however, and the model additionally introduces the idea of colour screening to regularise the $p_{\perp} \rightarrow 0$ divergence. The concept of a perturbative cross section is based on the assumption of free incoming states, which is not the case when partons are confined in colour-singlet hadrons. One therefore expects a colour charge to be screened by the presence of nearby anti-charges; that is, if the typical charge separation is $d$, gluons with a transverse wavelength $\sim 1 / p_{\perp}>d$ are no longer able to resolve charges individually, leading to a reduced effective coupling. This is introduced by reweighting the interaction cross section such that it is regularised according to

$$
\frac{\mathrm{d} \hat{\sigma}}{\mathrm{d} p_{\perp}^{2}} \propto \frac{\alpha_{\mathrm{S}}^{2}\left(p_{\perp}^{2}\right)}{p_{\perp}^{4}} \rightarrow \frac{\alpha_{\mathrm{s}}^{2}\left(p_{\perp_{0}}^{2}+p_{\perp}^{2}\right)}{\left(p_{\perp_{0}}^{2}+p_{\perp}^{2}\right)^{2}},
$$


where $p_{\perp 0}$ (related to $1 / d$ above) is now a free parameter in the model.

This parameter has an energy dependence, and the ansatz used is that it scales in a similar manner to the total cross section, i.e. driven by an effective power related to the Pomeron intercept [54, which in turn could be related to the small- $x$ behaviour of parton densities. This leads to a scaling

$$
p_{\perp 0}\left(E_{\mathrm{CM}}\right)=p_{\perp 0}^{\mathrm{ref}} \times\left(\frac{E_{\mathrm{CM}}}{E_{\mathrm{CM}}^{\mathrm{ref}}}\right)^{E_{\mathrm{CM}}^{\mathrm{pow}}}
$$

where $E_{\mathrm{CM}}^{\mathrm{ref}}$ is some convenient reference energy and $p_{\perp 0}^{\mathrm{ref}}$ and $E_{\mathrm{CM}}^{\mathrm{pow}}$ are parameters to be tuned to data.

\subsection{Hadronic matter distribution}

In the original MPI framework of [1], events are characterised by a varying impact parameter, $b$, representing a classical distance of closest approach between the two incoming hadrons. The hadronic matter is assumed to have a spherically symmetric distribution, taken to be the same for all parton species and momenta. The time-integrated overlap between the two incoming matter distributions at an impact parameter, $b$, is given by

$$
\tilde{\mathcal{O}}(b)=\int \mathrm{d} t \int \mathrm{d}^{3} x \rho(x, y, z) \rho\left(x, y, z-\sqrt{b^{2}+t^{2}}\right)
$$

where the $\rho$ 's give the matter distributions after a scale change to take into account the boosted nature of the hadrons. There are currently three different matter profiles available:

1) Single Gaussian: a simple Gaussian with no free parameters

$$
\rho(r) \propto \exp \left(-r^{2}\right)
$$

2) Double Gaussian: a core region, radius $a_{2}$, contains a fraction $\beta$ of the total hadronic matter, embedded in a larger hadron of radius $a_{1}$. The default parameters for this profile are $a_{2} / a_{1}=0.4$ and $\beta=0.5$

$$
\rho(r) \propto(1-\beta) \frac{1}{a_{1}^{3}} \exp \left(-\frac{r^{2}}{a_{1}^{2}}\right)+\beta \frac{1}{a_{2}^{3}} \exp \left(-\frac{r^{2}}{a_{2}^{2}}\right) .
$$

3) Overlap function: $\tilde{\mathcal{O}}(b)$, rather than $\rho(r)$, is parameterised by a single parameter, $p$. When $p=2$, this gives the single Gaussian behaviour, while when $p=1$, results are similar to the default double Gaussian behaviour

$$
\tilde{\mathcal{O}}(b) \propto \exp \left(-b^{p}\right)
$$

In what follows, we relax the assumption that this distribution remains the same for all momenta, such that the wavefunction for small- $x$ partons is broader in spatial extent than for large- $x$ ones. In particular, a form

$$
\rho(r, x) \propto \frac{1}{a^{3}(x)} \exp \left(-\frac{r^{2}}{a^{2}(x)}\right)
$$




$$
a(x)=a_{0}\left(1+a_{1} \ln \frac{1}{x}\right)
$$

is chosen, where $x$ represents the momentum fraction of the parton being probed within the hadron, $a_{0}$ is a constant to be tuned according to the non-diffractive cross section (detailed below) and $a_{1}$ is a free parameter. When $a_{1}=0$, the single Gaussian profile is recovered. With this matter profile, the time-integrated overlap is given by

$$
\tilde{\mathcal{O}}\left(b, x_{1}, x_{2}\right)=\frac{1}{\pi} \frac{1}{a^{2}\left(x_{1}\right)+a^{2}\left(x_{2}\right)} \exp \left(-\frac{b^{2}}{a^{2}\left(x_{1}\right)+a^{2}\left(x_{2}\right)}\right)
$$

where the normalisation has been chosen such that

$$
\int \tilde{\mathcal{O}}\left(b, x_{1}, x_{2}\right) \mathrm{d}^{2} b=1
$$

\subsection{Impact parameter framework}

Within the framework, the number of interactions is assumed to be distributed according to a Poissonian distribution. If $\bar{n}(b)$ gives the average number of interactions when two hadrons pass each other with an impact parameter $b$, the probability that there is at least one interaction is given by

$$
P_{\text {int }}(b)=1-e^{-\bar{n}(b)} .
$$

This gives the requirement for an event to be produced in the first place. The average number of interactions per event at impact parameter $b$ is therefore given by

$$
\left.\bar{n}(b)\right|_{n \neq 0}=\frac{\bar{n}(b)}{P_{\mathrm{int}}(b)} .
$$

When integrated over all impact parameters, the relation $\langle n\rangle=\sigma_{\text {hard }} / \sigma_{\mathrm{ND}}$ (Sec. 2) must still hold, giving

$$
\langle n\rangle=\frac{\left.\int \bar{n}(b)\right|_{n \neq 0} P_{\text {int }}(b) \mathrm{d}^{2} b}{\int P_{\text {int }}(b) \mathrm{d}^{2} b}=\frac{\int \bar{n}(b) \mathrm{d}^{2} b}{\int\left(1-e^{-\bar{n}(b)}\right) \mathrm{d}^{2} b}=\frac{\sigma_{\text {hard }}}{\sigma_{\mathrm{ND}}} .
$$

Defining the shorthand $X=\left(x_{1}, x_{2}, p_{\perp}^{2}\right)$ and $\mathrm{d} X=\mathrm{d} x_{1} \mathrm{~d} x_{2} \mathrm{~d} p_{\perp}^{2}, \sigma_{\text {hard }}$ may now be written as

$$
\sigma_{\text {hard }}=\int \mathrm{d} X \frac{\mathrm{d} \sigma}{\mathrm{d} X}=\iint \mathrm{d} X \mathrm{~d}^{2} b \frac{\mathrm{d} \sigma}{\mathrm{d} X} \tilde{\mathcal{O}}\left(b, x_{1}, x_{2}\right)
$$

where eq. (12) has been used to associate an impact-parameter profile with each $X$ coordinate. Here, $\mathrm{d} \sigma / \mathrm{d} X$ gives the convolution of PDF factors and the (regularised) hard partonic cross section

$$
\frac{\mathrm{d} \sigma}{\mathrm{d} X}=\left.f_{1}\left(x_{1}, p_{\perp}^{2}\right) f_{2}\left(x_{2}, p_{\perp}^{2}\right) \frac{\mathrm{d} \hat{\sigma}}{\mathrm{d} p_{\perp}^{2}}\right|_{\mathrm{reg}} .
$$

Comparing with eq. (15), this gives the average number of interactions at an impact parameter $b$ to be

$$
\bar{n}(b)=\int \mathrm{d} X \frac{\mathrm{d} \sigma}{\mathrm{d} X} \tilde{\mathcal{O}}\left(b, x_{1}, x_{2}\right) .
$$


One can now give a geometrical interpretation to $\sigma_{\text {hard }}$ and $\sigma_{\mathrm{ND}}$

$$
\begin{aligned}
\sigma_{\text {hard }} & =\int \bar{n}(b) \mathrm{d}^{2} b, \\
\sigma_{\mathrm{ND}} & =\int P_{\text {int }}(b) \mathrm{d}^{2} b=\int\left(1-e^{-\bar{n}(b)}\right) \mathrm{d}^{2} b,
\end{aligned}
$$

such that eq. (15) is fulfilled. This determines the value of $a_{0}$ as follows. Eq. (19) fixes the total area of $\bar{n}(b)$, within the constraint that it is possible to have either a large width $a_{0}$ and a small height $\bar{n}(0)$, or the other way around. In the former case, $1-\exp (-\bar{n}(0)) \approx \bar{n}(0)$, giving $\sigma_{\mathrm{ND}} \approx \sigma_{\text {hard }}$. In the latter, strong saturation effects lead to $1-\exp (-\bar{n}(0)) \ll \bar{n}(0)$, giving $\sigma_{\mathrm{ND}} \ll \sigma_{\text {hard }}$. The saturation corrections increase monotonically with $\bar{n}(0)$ and so a unique solution for $a_{0}$ is defined. This is studied further in Sec. 3.1.

\subsection{Impact parameter selection}

In picking the hardest interaction in an event, $p_{\perp 1}^{2}$, the naive probability for a collision must be multiplied by the probability that there were no harder ones at scales $p_{\perp}^{2}>p_{\perp 1}^{2}$. Using the notation

$$
\bar{n}(b)=\int \frac{\mathrm{d} \bar{n}(b)}{\mathrm{d} p_{\perp}^{2}} \mathrm{~d} p_{\perp}^{2},
$$

the total probability distribution is now

$$
\frac{\mathrm{d} P_{\text {hardest }}}{\mathrm{d}^{2} b \mathrm{~d} p_{\perp 1}^{2}}=\frac{\mathrm{d} \bar{n}(b)}{\mathrm{d} p_{\perp 1}^{2}} \exp \left(-\int_{p_{\perp 1}^{2}}^{s / 4} \frac{\mathrm{d} \bar{n}(b)}{\mathrm{d} p_{\perp}^{2}} \mathrm{~d} p_{\perp}^{2}\right) .
$$

One possible way of generating events according to this distribution is through trial interactions, similar to e.g. trial showers in CKKW-L [55], in the following way. If the evaluation of the Sudakov factor is temporarily deferred, then

$$
\frac{\mathrm{d} P_{\text {hardest }}}{\mathrm{d} p_{\perp 1}^{2}}=\int \mathrm{d}^{2} b \frac{\mathrm{d} \bar{n}(b)}{\mathrm{d} p_{\perp 1}^{2}}=\left.\iint \mathrm{d} x_{1} \mathrm{~d} x_{2} f_{1}\left(x_{1}, p_{\perp 1}^{2}\right) f_{2}\left(x_{2}, p_{\perp 1}^{2}\right) \frac{\mathrm{d} \hat{\sigma}}{\mathrm{d} p_{\perp 1}^{2}}\right|_{\text {reg }} .
$$

$p_{\perp 1}^{2}, x_{1}$ and $x_{2}$ may then be picked according to the above distribution, before an impact parameter $b$ is selected according to $\tilde{\mathcal{O}}\left(b, x_{1}, x_{2}\right) \mathrm{d}^{2} b$. The scale of a trial MPI interaction, $p_{\perp 2}^{2}$, may then be generated for this $b$ value, as described in the next section. It is important to note that the $p_{\perp_{2}}^{2}$ evolution is started from the kinematical limit, $s / 4$, as for an event with no previous interaction. If $p_{\perp_{2}}^{2}<p_{\perp_{1}}^{2}$, then $p_{\perp_{1}}^{2}$ and $b$ are accepted, else the selection procedure must restart from the beginning.

The above provides a prescription for generating an inclusive sample of non-diffractive events (hereafter referred to as minimum bias), but can also be used to generate the MPI activity accompanying a pre-given hard process. This is simplest in the case where the hard process in question is already part of the set of processes contained in $\mathrm{d} \sigma / \mathrm{d} X$. Here, $p_{\perp_{1}}^{2}$ is provided by this hard process, and the MPI framework should not generate any interactions at higher scales, or else one would double count. Given a hard process at a scale $p_{\perp 1}^{2}, b$ can be selected from $\tilde{\mathcal{O}}\left(b, x_{1}, x_{2}\right) \mathrm{d}^{2} b$, and then retained with a probability equal to the Sudakov of eq. (22). Again, trial interactions are a possible way to generate this Sudakov factor. 
When the hard process is not contained in $\mathrm{d} \sigma / \mathrm{d} X$, such as $\mathrm{Z}^{0}$ production, the MPI framework can begin evolution at the kinematical limit without any risk of double counting. For this discussion, noting that $p_{\perp}$ is intended as a measure of hardness, we assume a scale such as $\hat{s}$ to be a reasonable choice for this process. One choice that must be made relates to which interaction is used in selecting the impact parameter for an event. If we decide that it is always the hardest interaction in an event, it should be remembered that there is now the possibility that this is an MPI, although this is rather unlikely for a process already picked to be hard.

When the pre-given hard process has a scale above $10-20 \mathrm{GeV}$, the Sudakov of eq. (22) will be close to unity, meaning that $b$ can be directly selected from $\tilde{\mathcal{O}}\left(b, x_{1}, x_{2}\right) \mathrm{d}^{2} b$ and any ambiguity will be minor. For hard processes around these scales, the correct procedure is less clear. One choice would be to retain the selection according to $\tilde{\mathcal{O}}\left(b, x_{1}, x_{2}\right) \mathrm{d}^{2} b$ only, while another would be to additionally apply a Sudakov weight to the selection.

In the hard process studies that follow, the impact parameter is always selected according to the hard process (not necessarily the hardest interaction, as above), and this selection is not weighted with a Sudakov. As above, for hard processes above $10-20 \mathrm{GeV}$, these choices do not greatly affect the outcome. With the single Gaussian, double Gaussian and overlap matter profiles, the MPI framework will give exactly the same impact parameter profile for e.g. a $1 \mathrm{TeV} Z^{\prime}$ as for $Z^{0}$ production. The amount of MPI activity is modified by longitudinal correlations, however, such that the $1 \mathrm{TeV} Z^{\prime}$ will, on average, have less activity. The new matter profile, with its varying width, instead, dynamically changes to give more underlying activity for the more massive state, given the differing $x$ values that enter.

\subsection{Subsequent evolution}

Once $b$ has been fixed, as in the previous section, the remaining sequence of multiple interactions must be generated according to

$$
\frac{\mathrm{d} P}{\mathrm{~d} p_{\perp i}}=\frac{\mathrm{d} \bar{n}(b)}{\mathrm{d} p_{\perp i}} \exp \left(-\int_{p_{\perp i}}^{p_{\perp i-1}} \frac{\mathrm{d} \bar{n}(b)}{\mathrm{d} p_{\perp}^{2}} \mathrm{~d} p_{\perp}^{2}\right) .
$$

This can be achieved through the veto algorithm [3], as follows. Temporarily neglecting the impact parameter dependence, an overestimate of the form

$$
\left.\iint \mathrm{d} x_{1} \mathrm{~d} x_{2} f_{1}\left(x_{1}, p_{\perp}^{2}\right) f_{2}\left(x_{2}, p_{\perp}^{2}\right) \frac{\mathrm{d} \hat{\sigma}}{\mathrm{d} p_{\perp}^{2}}\right|_{\text {reg }} \leq \frac{N}{\left(p_{\perp}^{2}+r p_{\perp 0}^{2}\right)^{2}}
$$

can be used, where $r$ and $N$ are tunable factors; the former to help flatten the correction ratio, to improve generation efficiency, and the latter to ensure that the overestimate sits above the cross section over the entire phase space. With the impact parameter dependence present, an additional factor, giving the maximum of the overlap distribution is introduced

$$
\begin{gathered}
\tilde{\mathcal{O}}\left(b, x_{1}, x_{2}\right) \leq \tilde{\mathcal{O}}_{\max }(b)=\frac{1}{2 \pi a_{0}^{2}} \exp \left(-\frac{b^{2}}{2 a_{\text {max }}^{2}}\right), \\
a_{\max }=a_{0}\left(1+a_{1} \ln \frac{1}{x_{\min }}\right)
\end{gathered}
$$


(a)

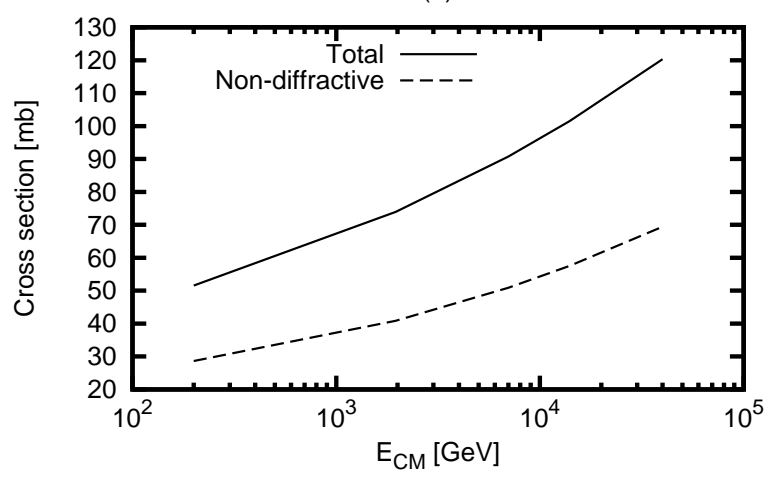

(b)

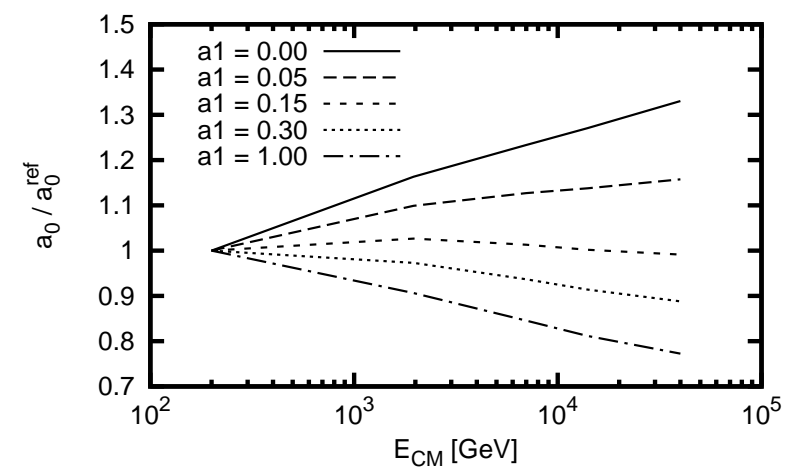

Figure 1: (a) The rise of the total and non-diffractive pp cross section with energy, and (b) the ratio $a_{0}\left(E_{\mathrm{CM}}\right) / a_{0}(200 \mathrm{GeV})$, over the same energy range, for a set of different $a_{1}$ values

where the first factor gives the maximum height of the distribution, while the exponential width is dictated by smallest $x$ values reached. This then gives a total overestimate

$$
\frac{\mathrm{d} \bar{n}(b)}{\mathrm{d} p_{\perp}^{2}} \leq \frac{N \tilde{\mathcal{O}}_{\max }(b)}{\left(p_{\perp}^{2}+r p_{\perp 0}^{2}\right)^{2}},
$$

giving a uniform overestimation for all $x_{1}$ and $x_{2}$

$$
\int \tilde{\mathcal{O}}_{\max }(b) \mathrm{d}^{2} b=\frac{a_{\max }^{2}}{a_{0}^{2}} .
$$

Eq. (28) is inserted into eq. (24) to pick the next $p_{\perp}$ scale. The additional acceptance weight for this interaction is now given by

$$
\frac{\tilde{\mathcal{O}}\left(b, x_{1}, x_{2}\right)}{\tilde{\mathcal{O}}_{\max }(b)}=\frac{2 a_{0}^{2}}{a^{2}\left(x_{1}\right)+a^{2}\left(x_{2}\right)} \exp \left(\frac{b^{2}}{2 a_{\max }^{2}}-\frac{b^{2}}{a^{2}\left(x_{1}\right)+a^{2}\left(x_{2}\right)}\right) \text {. }
$$

In case of failure, the evolution in $p_{\perp}$ is continued downwards from the rejected $p_{\perp}$ value.

\section{Results}

\subsection{Growth of the total cross section}

In principle, $a_{1}$, as introduced so far, is a free parameter. If, however, as suggested earlier, the wider profile of low- $x$ partons is to account for the growth of the total cross section (or the inelastic non-diffractive one, as in this model), then it can be constrained by the requirement that $a_{0}$ should be independent of energy.

The total cross section is taken from a Donnachie-Landshoff parameterisation [54. It is also necessary to break this down into elastic, diffractive and non-diffractive components, which is done based on a parameterisation incorporating empirical corrections such that the elastic and diffractive cross sections do not exceed the total at higher energies [56]. In 
(a)

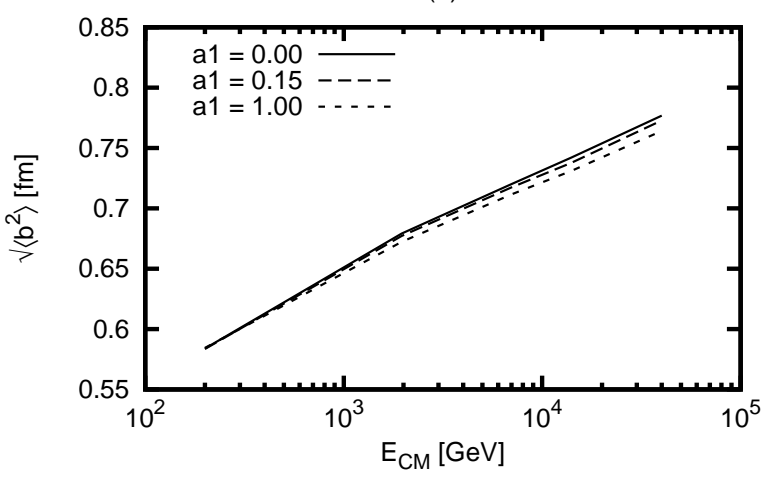

(b)

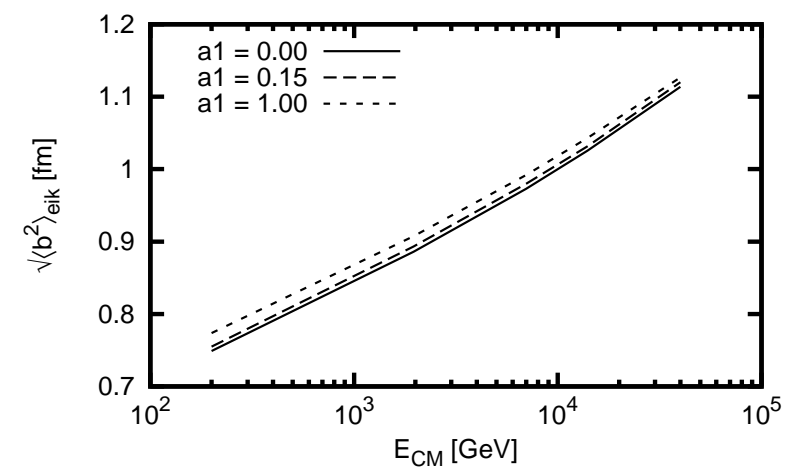

Figure 2: Root-mean-squared value of (a) $\bar{n}$ and (b) $P_{\text {int }}$ as a function of the centre-of-mass energy

all that follows, we will deal explicitly with pp collisions. The assumed rise of the total and non-diffractive cross sections are shown as a function of centre-of-mass energy in Fig. 11a.

In the calculation of $a_{0}, \mathrm{~d} \sigma / \mathrm{d} X$ enters, giving a dependence on PDFs and the $p_{\perp 0}$ used to regularise the cross section. In all that follows, the parameters of Tune $4 \mathrm{C}$ [9], a tune to early LHC data, are used. It is the relative variation of $a_{0}$ as a function of energy that is of interest here, and in Fig. 1 b the ratio $a_{0}\left(E_{\mathrm{CM}}\right) / a_{0}(200 \mathrm{GeV})$ is shown over the same range of energies, for a set of different $a_{1}$ values. A value of $a_{1}=0.15$ gives an $a_{0}$ that is relatively stable across this energy range.

As discussed in Sec. 2.2, through the eikonalisation procedure and tuning of the $a_{0}$ parameter, the width of the matter profile has an absolute meaning, related to the size of the incoming hadron. As $a_{1}$ is increased from zero, $\bar{n}(b)$, after integration over $x$ values, is higher both at small and large $b$ values, and smaller at intermediate $b$ values, such that the total area is conserved. In some ways the shape is similar to what one would expect from a double Gaussian matter profile, where the central core of matter would tend to push the distribution up at small $b$, while the tail would also have a larger content, due to the peripheral component.

Before eikonalisation, and given that the form of eq. (9) stretches out to infinity, a simple measure is given by the RMS value of $\bar{n}(b)$

$$
\left\langle b^{2}\right\rangle=\frac{\int b^{2} \bar{n}(b) \mathrm{d}^{2} b}{\int \bar{n}(b) \mathrm{d}^{2} b}=\frac{1}{\sigma_{\text {hard }}} \int b^{2} \bar{n}(b) \mathrm{d}^{2} b .
$$

The variation of $\left\langle b^{2}\right\rangle$ with energy is shown in Fig. 2a. The slope slightly decreases with the rise of $a_{1}$, dependent on the enhancement of $\bar{n}(b)$ at low $b$ relative to high.

Assuming a single Gaussian matter profile, the width, $a$, needed to give the same $\left\langle b^{2}\right\rangle$ is given by $a=\sqrt{3\left\langle b^{2}\right\rangle} / 2$, noting that the RMS radius is defined in 3 rather than 2 dimensions. This gives values beneath the conventional charge RMS radius. We do not study this further here, but do note that the eikonalisation procedure used ignores any contribution from diffractive components, also noted below.

The same quantity, after the eikonalisation procedure can also be obtained from

$$
\left\langle b^{2}\right\rangle_{\text {eik }}=\frac{\int b^{2} P_{\text {int }}(b) \mathrm{d}^{2} b}{\int P_{\text {int }}(b) \mathrm{d}^{2} b}=\frac{1}{\sigma_{\mathrm{ND}}} \int b^{2} P_{\text {int }}(b) \mathrm{d}^{2} b .
$$


The variation of this quantity with energy is shown in Fig. $2 \mathrm{~b}$. After eikonalisation, the component of $\bar{n}(b)$ at large $b$ becomes more important, leading to an increase as $a_{1}$ grows from zero, due to the contribution of low- $x$ partons.

A final consistency check is provided by the standard eikonal formulae [28], providing a relation between the total and inelastic cross sections

$$
\begin{aligned}
& \sigma_{\text {inel }}=\int \mathrm{d}^{2} b\left(1-e^{2 \chi(b)}\right), \\
& \sigma_{\text {tot }}=2 \int \mathrm{d}^{2} b\left(1-e^{\chi(b)}\right) .
\end{aligned}
$$

From the former equation, we can identify the eikonal function $\chi(b)=\bar{n}(b) / 2$. Using the latter equation to calculate the total cross section, the result is consistently below the total cross section of Fig. 1a by around $10-20 \%$. As noted above, the diffractive component has been ignored in the above framework, and is a potential source for these deviations, including the low $\left\langle b^{2}\right\rangle$ values noted previously.

\subsection{Hard processes}

In what follows, comparisons are made between the different hadronic matter distributions (Sec. 2.1). For impact parameter distributions, results are presented in terms of $b_{\mathrm{MPI}}^{\mathrm{norm}}=$ $b / b_{\text {avg, }}$ where

$$
b_{\text {avg }}=\frac{\int b P_{\text {int }}(b) \mathrm{d}^{2} b}{\int P_{\text {int }}(b) \mathrm{d}^{2} b}=\frac{1}{\sigma_{\mathrm{ND}}} \int b P_{\text {int }}(b) \mathrm{d}^{2} b,
$$

such that the average value is unity for minimum-bias events. Also of interest is the enhancement factor associated with each interaction, $\tilde{\mathcal{O}}\left(b, x_{1}, x_{2}\right)$ of eq. (18). This is also normalised such that the average is unity for the hard process in minimum-bias events, $e_{\text {hard }}^{\text {norm }}=\tilde{\mathcal{O}}\left(b, x_{1}, x_{2}\right) / e_{\text {avg }}$, where

$$
e_{\text {avg }}=\frac{\int \bar{n}(b) P_{\text {int }}(b) \mathrm{d}^{2} b}{\int \bar{n}(b) \mathrm{d}^{2} b}=\frac{1}{\sigma_{\text {hard }}} \int \bar{n}(b) P_{\text {int }}(b) \mathrm{d}^{2} b,
$$

compensating for the fact that the average number of interactions is raised by removing the sample with no interactions.

As discussed in Sec. 2.3, those processes with final states which cannot be produced by the MPI framework will begin their $p_{\perp}$ evolution at the kinematical limit. Due to this, their impact parameter profiles will be picked directly according to $\tilde{\mathcal{O}}\left(b, x_{1}, x_{2}\right) \mathrm{d}^{2} b$, with no Sudakov weighting. This offers a direct way to examine the effects of the new matter profile in comparison to previous ones.

\subsection{1 $Z^{0}$ production}

In this section, the MPI accompanying $\mathrm{Z}^{0}$ (with no $\gamma^{*}$ interference) production is studied. The following matter profiles are compared:

SG: single Gaussian,

DG: double Gaussian with default parameters $a_{2} / a_{1}=0.4$ and $\beta=0.5$,

Overlap: overlap function with $p=1.5$,

Log: logarithmically $x$-dependent Gaussian with $a_{1}=0.15$. 
(a)

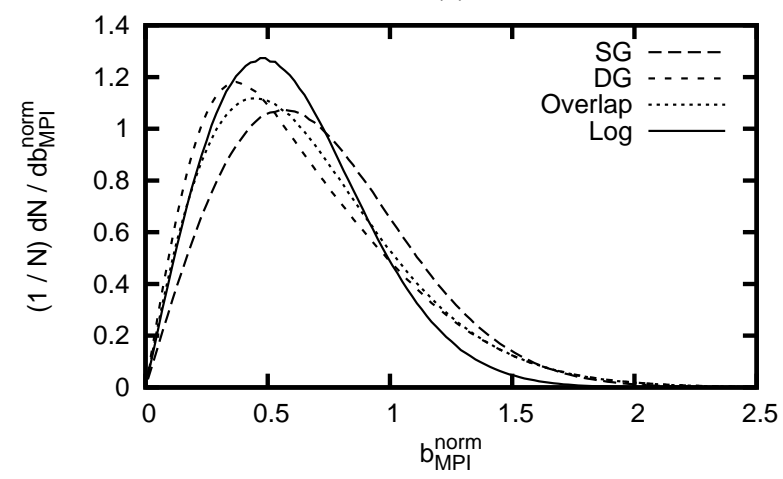

(c)

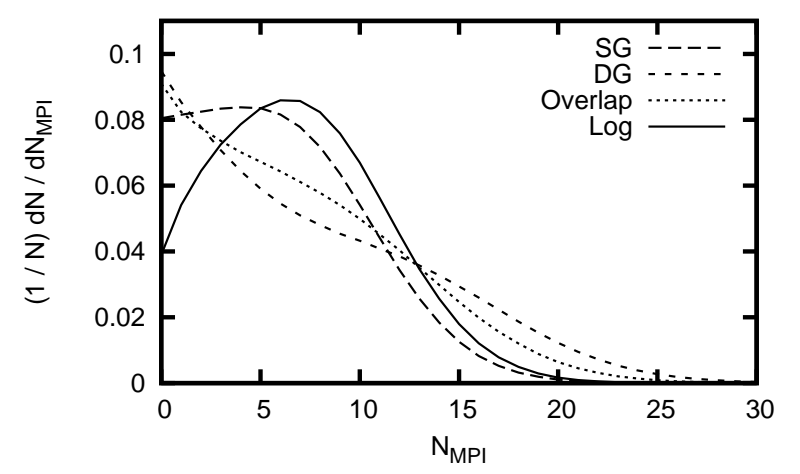

(b)

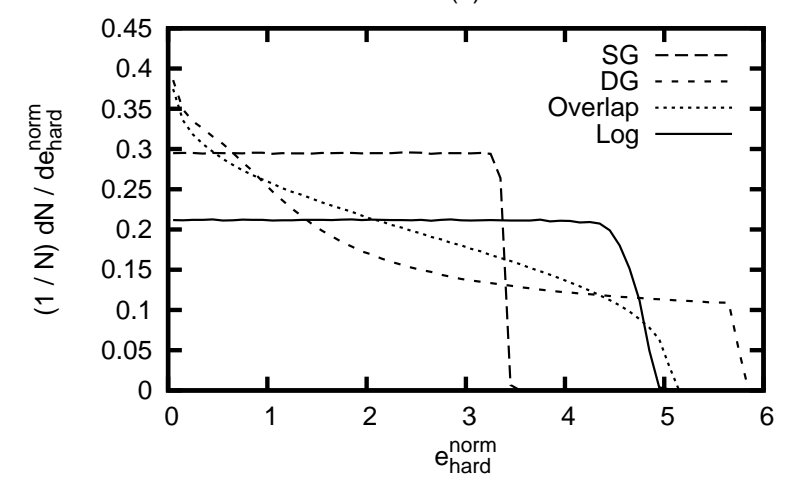

(d)

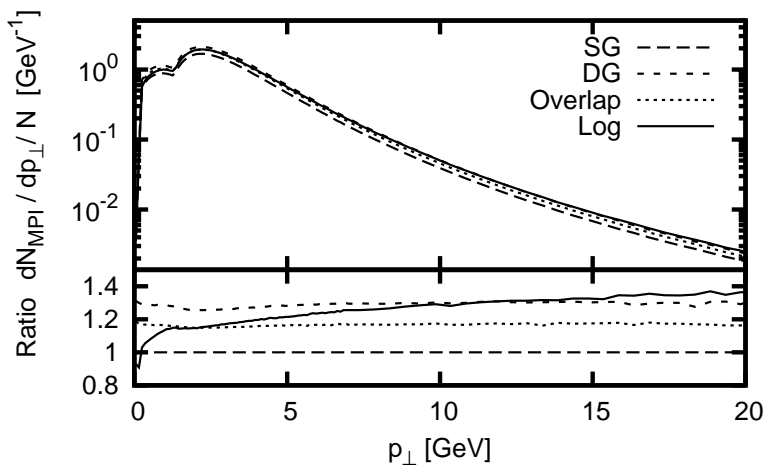

Figure 3: $\mathrm{Z}^{0}$ production in pp collisions at $7 \mathrm{TeV}$. (a) The impact parameter distribution, (b) enhancement factor of the hard interaction, (c) number of MPI and (d) inclusive $p_{\perp}$ spectrum of MPI per event. The ratio plot in (d) is normalised to the single Gaussian result

This process is studied in pp collisions at $\sqrt{s}=7 \mathrm{TeV}$. To study only the effects of the MPI model, parton showers are switched off. This does affect some longitudinal correlations; initial-state radiation, in particular, is in competition with MPI for momentum from the beams.

Fig. 3 3 shows the impact parameter distributions for these matter profiles. As outlined previously, in $\mathrm{Z}^{0}$ production, they are picked unmodified from $\tilde{\mathcal{O}}\left(b, x_{1}, x_{2}\right) \mathrm{d}^{2} b$, but shown normalised such that the average value would be unity for minimum bias events. Noting that $\mathrm{d}^{2} b \propto b \mathrm{~d} b$, it is possible to study the general features of $\tilde{\mathcal{O}}\left(b, x_{1}, x_{2}\right)$ itself. In the double Gaussian profile, relative to the single Gaussian, the central core of hadronic matter dominates at small $b$, giving larger overlap values, although with a faster fall-off. The peripheral Gaussian component then slows this fall-off, giving contributions out to larger $b$ values. The overlap scenario sits roughly between the single and double Gaussian distributions. The log profile is now an average of single Gaussian overlap functions, whose widths are determined by the combination of $x_{1}$ and $x_{2}$ values that contribute. These $x$ values give a narrower distribution than the single Gaussian case. At larger values of $a_{1}$, this distribution would become even more narrow. It is these features which directly give rise to the form of the impact parameter profile, shown in the figure. In particular, the log scenario is peaked at smaller values than the single Gaussian, but without a large tail out 
to high $b$ values, unlike the double Gaussian.

In Fig. 3b the distribution of the enhancement factor, $\tilde{\mathcal{O}}\left(b, x_{1}, x_{2}\right)$, is shown, again normalised such the average value would be unity for minimum bias events. It is noted that this distribution gives the overlap for those events where $b$ has already been selected according to $\tilde{\mathcal{O}}\left(b, x_{1}, x_{2}\right) \mathrm{d}^{2} b$. Formally, this is stated by $\mathrm{d} N / \mathrm{d} \tilde{\mathcal{O}}\left(b, x_{1}, x_{2}\right)=\mathrm{d} N / \mathrm{d} b * \mathrm{~d} b / \mathrm{d} \tilde{\mathcal{O}}\left(b, x_{1}, x_{2}\right)$, where $\mathrm{d} N / \mathrm{d} b \propto b \tilde{\mathcal{O}}\left(b, x_{1}, x_{2}\right)$. For the single Gaussian, where also $\mathrm{d} \tilde{\mathcal{O}}\left(b, x_{1}, x_{2}\right) / \mathrm{d} b \propto$ $b \tilde{\mathcal{O}}\left(b, x_{1}, x_{2}\right)$, the enhancement is flat. It stretches from 0 to $\pi$, as determined by the normalisation of eq. (11). The log profile is again an average of single Gaussians. The upper cutoff is determined by the combination of $x$ values that gives the lowest possible $a^{2}\left(x_{1}\right)+a^{2}\left(x_{2}\right)$. The width and shape of the fall-off is related to the range and relative rate of these $x$ combinations, respectively. The shape for the overlap scenario can similarly be calculated. For an overlap $\tilde{\mathcal{O}}(b)=k \exp \left(-b^{1.5}\right)$, this is given by $\mathrm{d} N / \mathrm{d} \tilde{\mathcal{O}}(b) \propto-\ln ^{1 / 3}(k / \tilde{\mathcal{O}}(b))$. It is not so easily calculated for the double Gaussian, but the shape of the distribution can be understood as its peripheral Gaussian component, stretching out to large $b$, giving the peaking behaviour as $e_{\text {hard }}^{\text {norm }} \rightarrow 0$.

It is perhaps the number of MPI accompanying $\mathrm{Z}^{0}$ production and their $p_{\perp}$ spectrum that are of more interest, since they directly influence physical observables. The distribution of the number of MPI per event is shown in Fig. 3k. For the single Gaussian, double Gaussian and overlap scenarios, the enhancement factor of the hard process is retained for the remaining sequence of MPI. Before taking into account energy-momentum conservation, the average number of MPI per event is directly proportional to the enhancement factor, with the actual number fluctuating around this mean value. The PDF rescaling then suppresses the high tail of this distribution, pushing events to smaller $N_{\text {MPI }}$. This does affect the overall shape of the curves, but it remains true that the widths of the $N_{\text {MPI }}$ distributions are essentially dictated by the widths of $e_{\text {hard }}^{\text {norm }}$ For the double Gaussian and overlap scenarios, the peaking behaviour as $e_{\text {hard }}^{\text {norm }} \rightarrow 0$ gives a similarly peaked distribution as $N_{\text {MPI }} \rightarrow 0$.

For the log profile, the narrower impact parameter gives rise to fewer events with small numbers of MPI. The tail, however, does not go out much beyond the single Gaussian, as the double Gaussian and overlap profiles do. This can be explained by the fact that the enhancement factor for the sequence of MPI is no longer fixed to the hard enhancement factor, but varies as a function of the $x$ values of each individual interaction.

The dominant process in MPI is $t$-channel gluon exchange. The parton-level process has no suppression at large- $x$ values, but is affected by PDF factors. Given the requirement that $\tau=x_{1} x_{2}<4 p_{\perp}^{2} / s$, as $p_{\perp}$ falls, the minimum $\tau$ also falls, opening up new regions of allowed $x$ values. From the small- $x$ peaking of the PDFs, one would expect that, on average, the $x$ values will fall as $p_{\perp}$ does. This, in turn, would lead to lower enhancement factors, as the partons become more smeared out in the proton.

The result of the above is visible in Fig. 3 $\mathrm{d}$, where the increase in the inclusive $p_{\perp}$ spectra of MPI is flat for the double Gaussian and overlap profiles, relative to the single Gaussian, but falls off towards low $p_{\perp}$ for the log scenario. The slope of this fall-off is affected by the impact parameter distribution, which, in turn, is determined by the $x$ values of the hard process. There are also additional PDF rescaling effects at play, but checks show that these are small. The change in shape of the absolute distributions at $\sim 2 \mathrm{GeV}$ is due to the freezing of the PDFs. Overall, then, the changing enhancement factor of MPI regulates the amount of MPI, affecting the tail of the log profile in Fig. 3 c. 


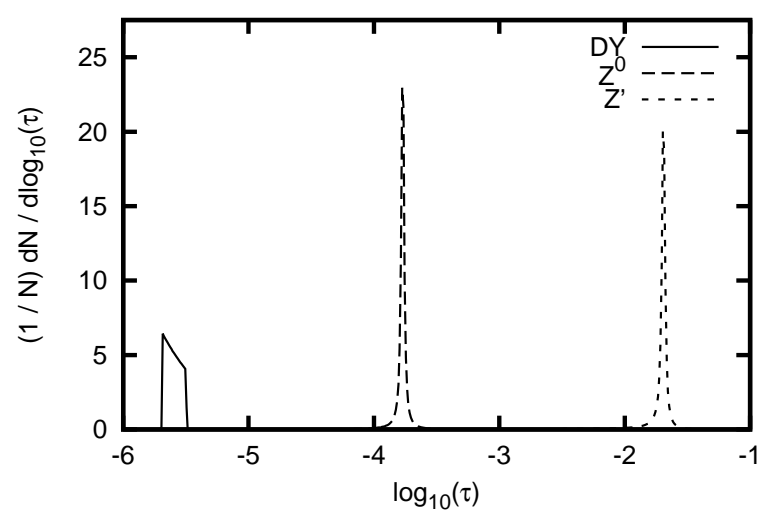

Figure 4: The $\tau$ distribution of low mass Drell-Yan (DY), $\mathrm{Z}^{0}$ and $\mathrm{Z}^{\prime}$ events in pp collisions at $\sqrt{s}=7 \mathrm{TeV}$

\subsection{Low mass Drell-Yan, $\mathrm{Z}^{0}$ and $\mathrm{Z}^{\prime}$ production}

The next step of our comparisons is to include other processes, again where there is no Sudakov involved, but for which the log profile will dynamically produce variations in the distributions. In particular, the following processes are used as templates to explore different well-defined $x$ ranges:

DY: low mass Drell-Yan $(10.0<\hat{m}<12.5 \mathrm{GeV})$,

$\mathbf{Z}^{\mathbf{0}}$ : as in the previous section,

$\mathbf{Z}^{\prime}$ : a $1 \mathrm{TeV} \mathrm{Z}^{\prime}$ resonance.

Again, we note that parton showers are not switched on here, which also take momentum from the beams, affecting PDF rescaling. For the log profile, it is the combination of $x_{1}$ and $x_{2}$ together in eq. (9) that is important. In Fig. 4, the $\tau=x_{1} x_{2}$ distribution of the three processes is shown, with each contained in a well defined region.

In Sec. 2.3, some discussion was given relating to the choice of impact parameter in hard processes, when its scale is in regions where the Sudakov of eq. (22) begins to vary away from unity, and where the chance of having a harder MPI also grows. The Drell-Yan process used here will be affected by these issues. We side step them here; this comparison is designed to highlight the effects of the log profile in certain $x$-ranges, without the additional complications of the Sudakov factor. We retain the decision to pick impact parameters according to the hard process and without any Sudakov weighting.

Fig. 5 shows the (a) impact parameter and (b) enhancement factor in the hard process per event, this time comparing the processes given above. These are also compared to the single Gaussian profile, which, as noted previously, gives the same results for these distributions for all three processes. In Fig. 6, the number of MPI is shown for (a) lowmass Drell-Yan, (b) $\mathrm{Z}^{0}$ and (c) $\mathrm{Z}^{\prime}$. Fig. 7 shows the ratio of the inclusive $p_{\perp}$ spectrum of MPI for the log profile to the single Gaussian result for the three processes.

All the features in these distributions can be understood in terms of the previous discussion of $\mathrm{Z}^{0}$ events. As expected, the impact parameter distributions become narrower as the $\tau$ range in question increases, leading to a wider distribution for the enhancement factor in the hard process. The Drell-Yan process gives very similar results to the single Gaussian for the impact parameter, hard enhancement factor and number of MPI distributions. The 
(a)

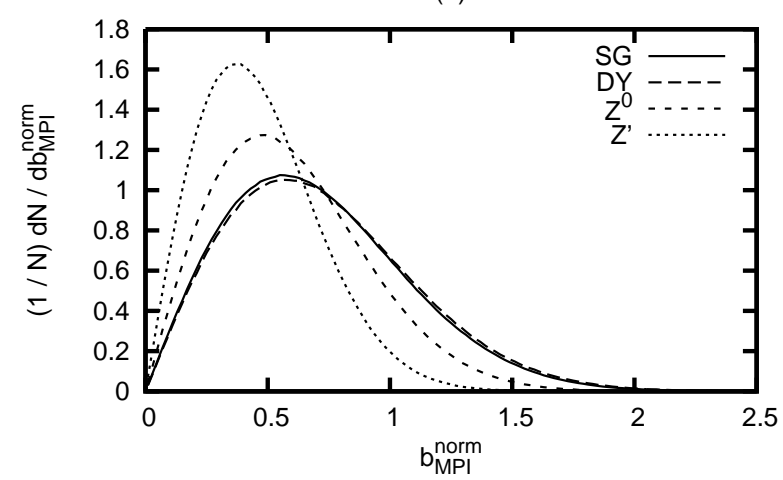

(b)

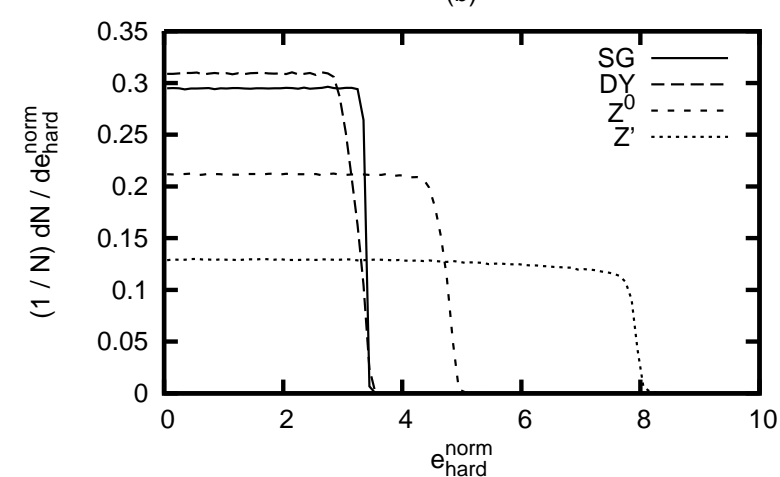

Figure 5: Low-mass Drell-Yan, $\mathrm{Z}^{0}$ and $\mathrm{Z}^{\prime}$ production in pp collisions at $\sqrt{s}=7 \mathrm{TeV}$. (a) The impact parameter distribution and (b) enhancement factor of the hard interaction per event. The single Gaussian distributions are identical between the three processes

(a) DY

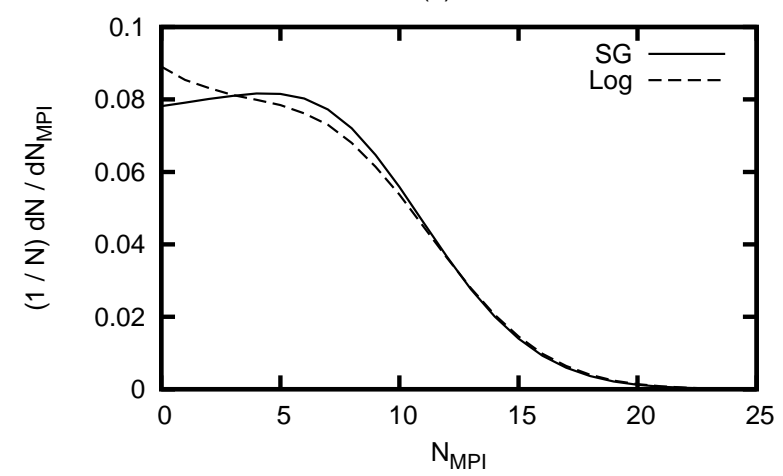

(b) $Z^{0}$

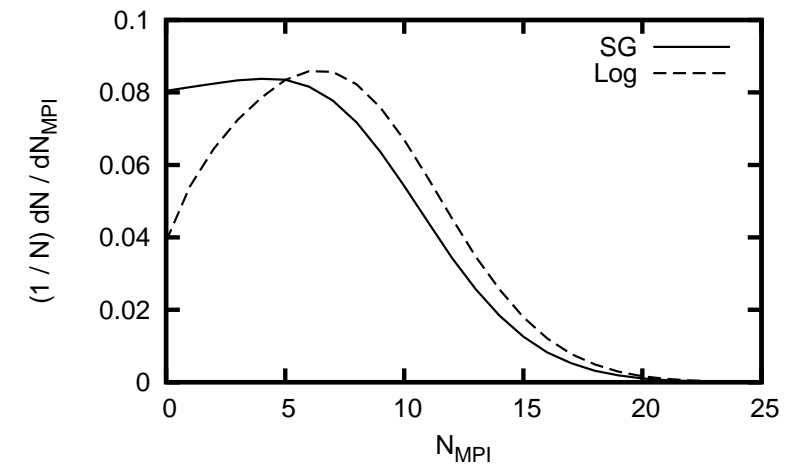

(c) Z'

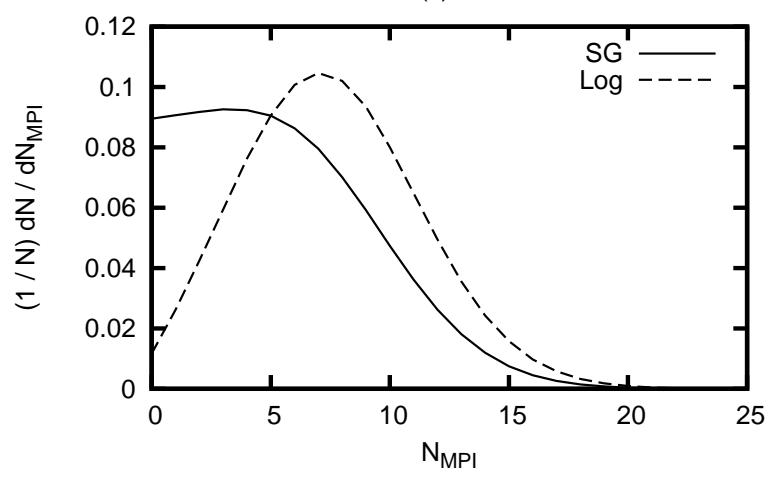

Figure 6: The number of MPI per event in (a) low-mass Drell-Yan, (b) $\mathrm{Z}^{0}$ and (c) $\mathrm{Z}^{\prime}$ production 


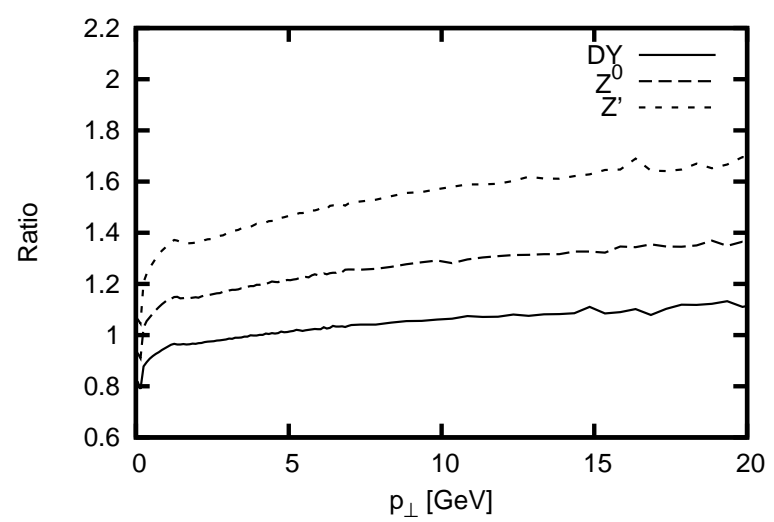

Figure 7: Ratio of the inclusive $p_{\perp}$ spectrum of MPI for the log profile to the single Gaussian result for the three different processes

$x$ values that contribute, in some sense, correspond to an average "hardness", with the same amount of activity above and below. All three processes have a similar endpoint in $N_{\text {MPI }}$, due to PDF rescaling. The even narrower impact parameter for $Z^{\prime}$ events further suppresses events with low $N_{\mathrm{MPI}}$, relative to $\mathrm{Z}^{0}$ production. The $p_{\perp}$ ratios of Fig. 7 all show an enhancement of high- $p_{\perp}$ activity relative to low, as expected. As the $x$ values of the hard process get larger, and the impact parameter profile narrower, the slope becomes steeper.

\subsection{Minimum bias}

We now move on to minimum-bias events. Here, there are additional correlations to consider, relative to the hard processes of the previous section. In particular, once a hard process has been selected, the subsequent MPI evolution will begin from this scale, meaning that high- $p_{\perp}$ events are likely to have more MPI, given the larger $p_{\perp}$ evolution range they have available. Low- $p_{\perp}$ events will also be biased towards larger impact parameters relative to high- $p_{\perp}$ ones, given the Sudakov weighting of eq. (22).

Again, we begin with the (a) impact parameter, (b) enhancement factor in the hard process and (c) number of MPI per event, now shown in Fig. 8, for the same four matter profiles used in $\mathrm{Z}^{0}$ production. The differences here are somewhat smaller than for the hard processes, so the ratios of the double Gaussian, overlap and log matter profiles to the single Gaussian are also given.

Both the impact parameter and enhancement factor distributions are now fixed, so that they have an average value of unity for all the different profiles. Any increase or decrease of these distributions over a given range must be compensated elsewhere. The shape of the impact parameter distributions are now directly dictated by $\bar{n}(b)$. For the log profile, it contains all the correlations brought about by the $x$-dependent width. Relative to the single Gaussian, the other three profiles show the same features; larger contributions at small and large $b$ values and a region of intermediate $b$ where it sits below. For the log profile, this is consistent with the shape of $\bar{n}(b)$ described in Sec. 3.1. Fig. 8 8 shows that the variation of the log profile, relative to the single Gaussian, is smaller than for the double Gaussian and overlap scenarios. 
(a)

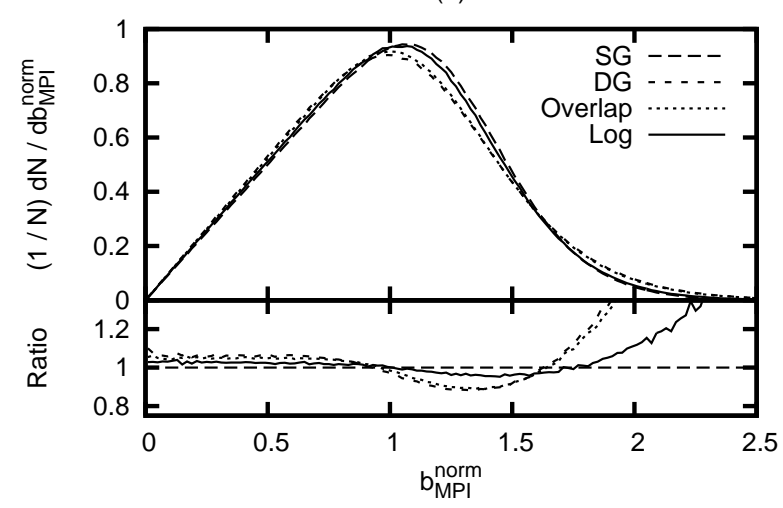

(b)

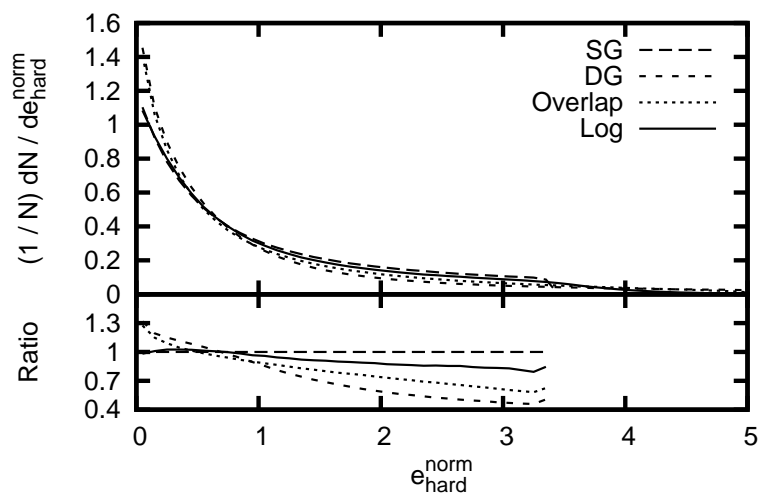

(c)

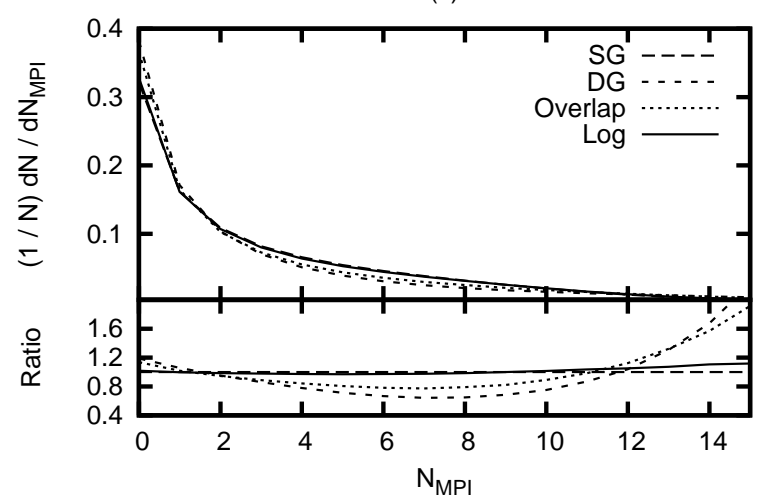

Figure 8: Minimum-bias events in pp collisions at $\sqrt{s}=7 \mathrm{TeV}$. (a) The impact parameter distribution, (b) enhancement factor of the hard interaction and (c) number of MPI per event. Ratio plots are normalised to the single Gaussian results

The overall shape of the $e_{\text {hard }}^{\text {norm }}$ distributions is given by the effect of the impact parameter profiles, which now vary as a function of the $p_{\perp}$ of the hard process, as noted above. Low$p_{\perp}$ events, which dominate, will be biased to higher impact parameters, giving the increase at low enhancement factors. Relative to the single Gaussian, the three other profiles all

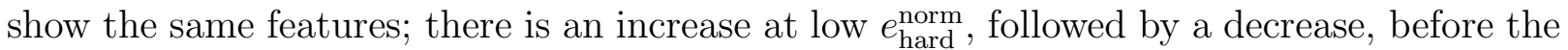
tails continue out beyond where the single Gaussian cuts off. These changes are a direct consequence of the changed impact parameter distributions.

For the $N_{\text {MPI }}$ distributions, the overall shape is now due to the correlation between $p_{\perp}$ and the number of MPI. On average, the more numerous low- $p_{\perp}$ events have less range of evolution, and therefore fewer MPI. The variation of the log profile, relative to the single Gaussian is small, but follows those of the double Gaussian and overlap; there is an increase at low $N_{\mathrm{MPI}}$, followed by a decrease, before again increasing in the tails. Again, these changes follow directly from the differences of the impact parameter distributions.

Finally, in Fig. 9, the $p_{\perp}$ distribution (a) of the hard process and (b) inclusive for subsequent MPI are shown. For the hard process, relative to the single Gaussian, the other three profiles give more activity at low $p_{\perp}$, relative to high. It becomes easier to have peripheral interactions involving small- $x$ partons, with the event containing no further activity. In the high- $p_{\perp}$ tails, the overlap is essentially saturated. There is a sharp rise as $p_{\perp} \rightarrow 0$ for the log profile. This change in shape is due to the freezing of the PDFs; in 
(a)

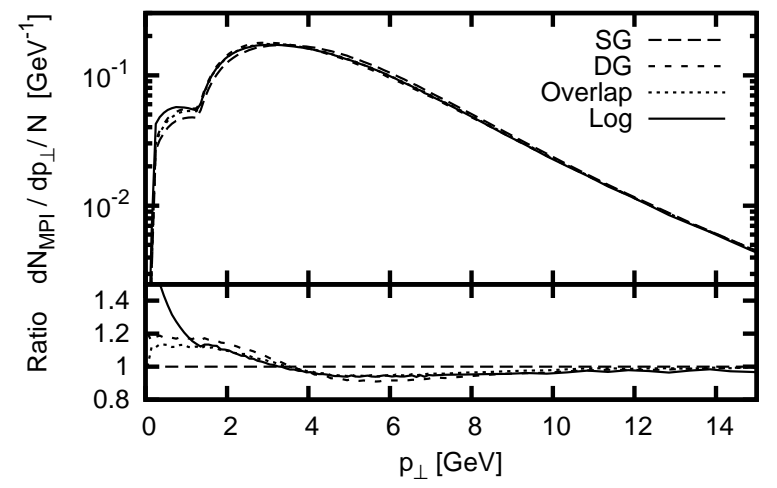

(b)

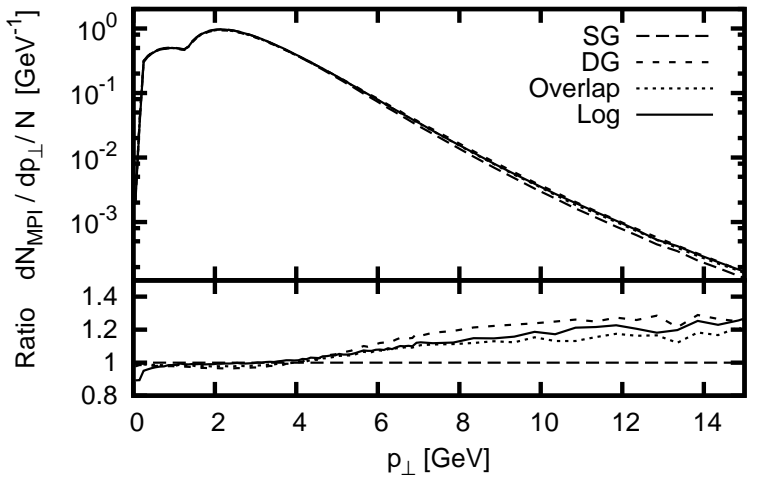

Figure 9: $p_{\perp}$ distribution (a) of the hard process and (b) inclusive for subsequent MPI in minimum bias events. Ratio plots are normalised to the single Gaussian result

these low- $p_{\perp}$ bins, there is no penalty to pay for taking higher $x$ values, up to this freezing point, resulting in extra contributions here. The inclusive $p_{\perp}$ spectra for the subsequent MPI now give exactly the opposite results to those of the hard process, such that, when they are summed together, they give back the unmodified $p_{\perp}$ spectrum of eq. (17), as they must.

\subsection{Minimum-bias and underlying-event studies}

We can examine the effect of this new matter profile on minimum-bias and underlying event studies. In particular, Tune $4 \mathrm{C}$, used also in the previous studies, offers an attractive starting point. This tune is based on a modification to a Tevatron tune, such that it is able to describe early LHC data. One of the features of this tune is a single Gaussian matter profile, which gives a reasonable match to both the rise of the underlying event as well as the width of charged multiplicity distributions in minimum-bias events.

In Fig. 10, the results of this tune are shown for (left-to-right, top-to-bottom):

1) ATLAS $\left(|\eta|<2.5, p_{\perp}>500 \mathrm{MeV}\right)$ INEL $>0$ minimum-bias dataset. Charged rapidity distribution in minimum-bias events at $\sqrt{s}=900 \mathrm{GeV}$ and $7 \mathrm{TeV}$ [57, 58]. The $900 \mathrm{GeV}$ data is taken from the online HEPDATA database, while the $7 \mathrm{TeV}$ data is taken from the corresponding reference.

2) As (1), but showing the charged multiplicity distributions. Errors are not included for the $7 \mathrm{TeV}$ data.

3) ATLAS $\left(|\eta|<2.5, p_{\perp}>500 \mathrm{MeV}\right)$ charged track based underlying event at $\sqrt{s}=$ $900 \mathrm{GeV}$ and $7 \mathrm{TeV}$ [59]. A charged track of $p_{\perp}>1 \mathrm{GeV}$ in the $\eta$ acceptance is required to trigger an event. Data and errors have been read off from the corresponding reference. Charged particle number density in the toward region.

4) As (3), but showing the sum- $p_{\perp}$ density in the toward region.

5) As (3), but showing the charged particle number density in the transverse region. 

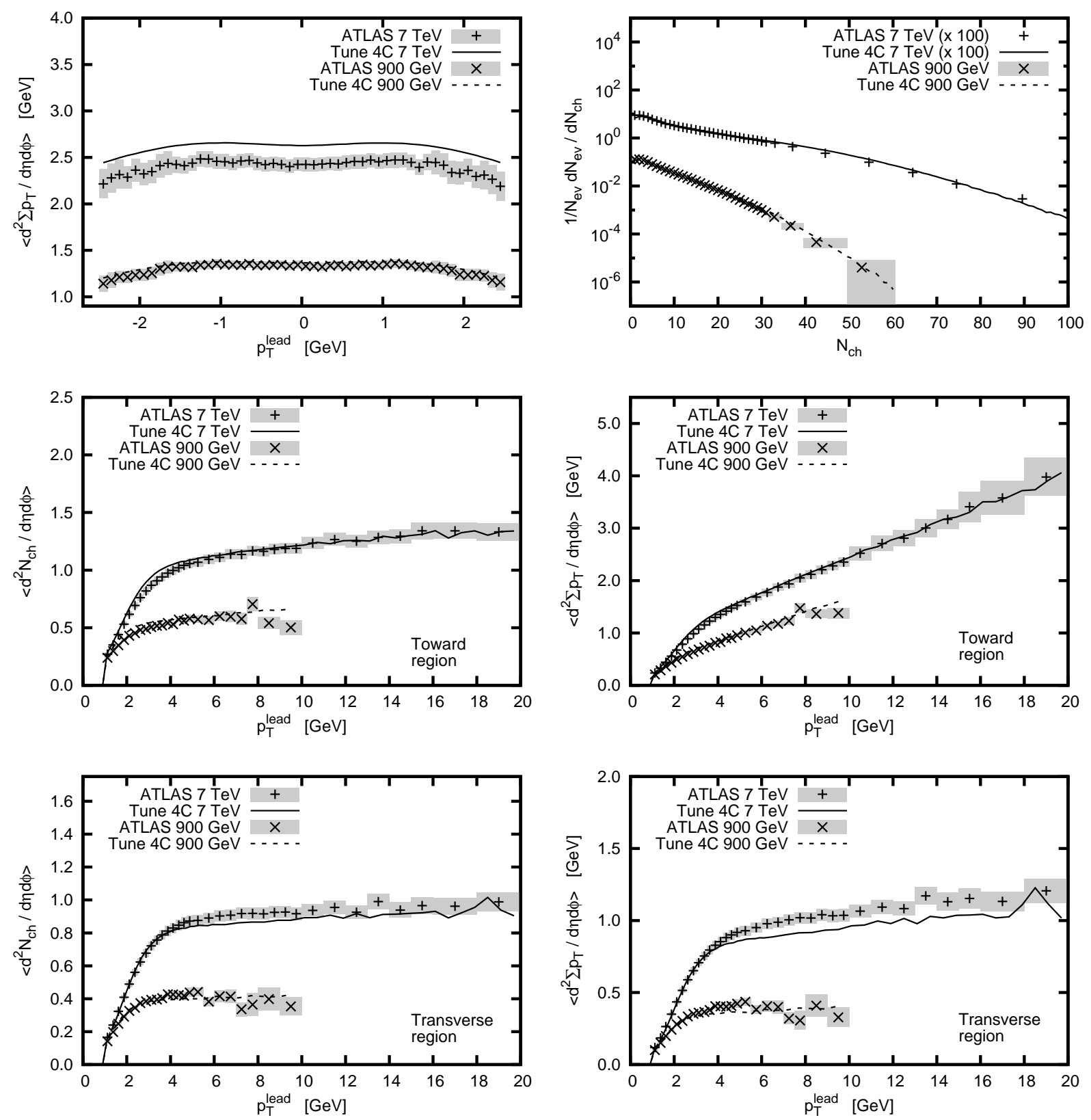

Figure 10: Tune 4C compared against early LHC data. Further details are given in the text

6) As (3), but showing the sum- $p_{\perp}$ density in the transverse region.

Where errors are shown, they represent the systematic and statistical errors summed in quadrature. Although the rise of the underlying event is too steep in the toward region, and activity in the transverse region is slightly too low, overall it gives a reasonable description of data.

The first step is to replace the single Gaussian with the logarithmically $x$-dependent matter profile with $a_{1}=0.15$. This change is made just in the non-diffractive component, while that used in diffractive framework for now remains a single Gaussian. Diffraction primarily impacts the low bins of the charged multiplicity distribution, and will not greatly 

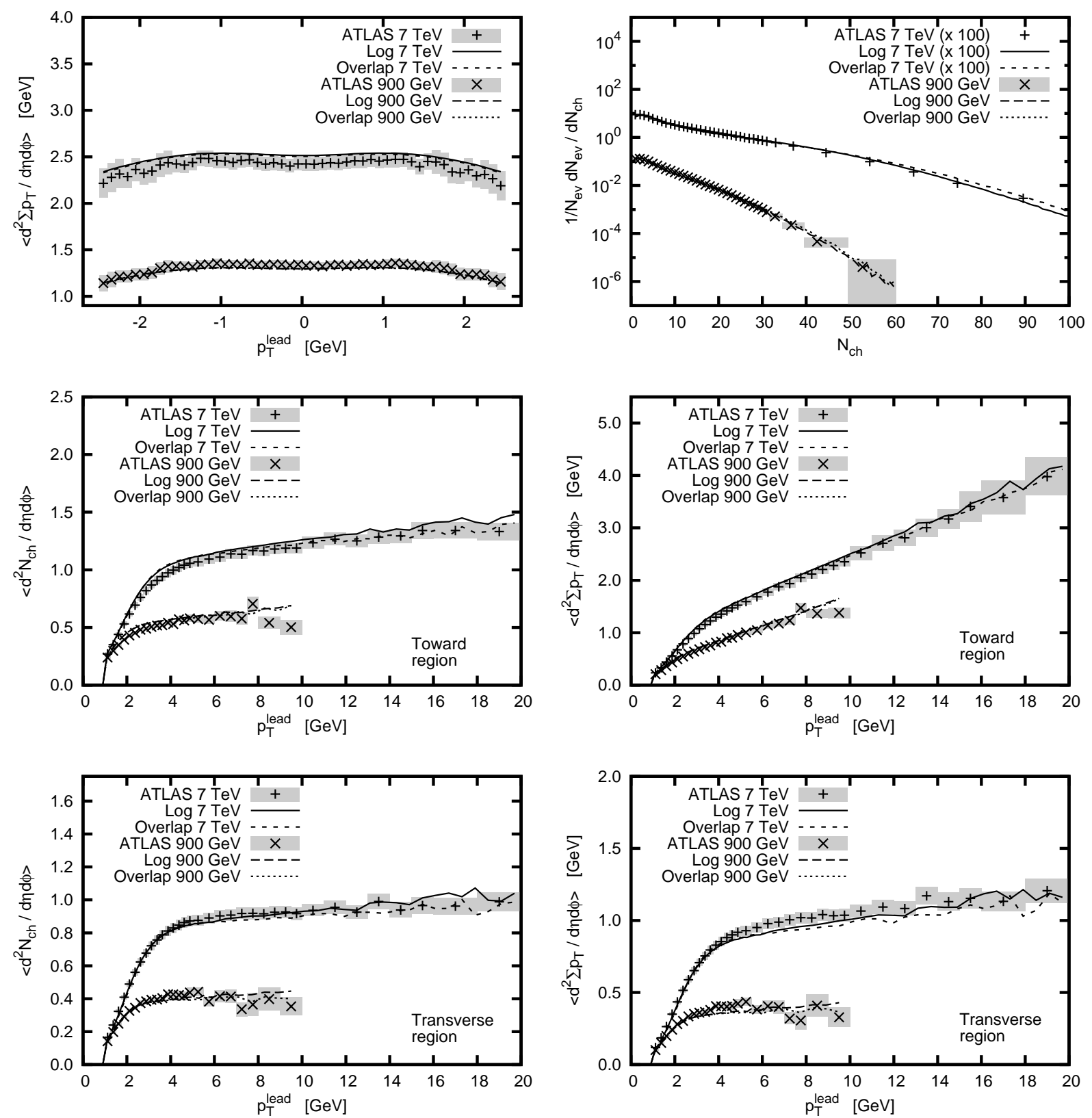

Figure 11: Tune $4 \mathrm{C}$, using the $\log$ profile, and with a raised $p_{\perp 0}$ in the MPI framework, compared against an overlap profile with $p=1.6$, also with a raised $p_{\perp 0}$, and LHC data

affect the results shown here. Just this change leads to a rise in the tail of the charged multiplicity distributions, with an increase in activity in all regions of the underlying event, as expected from the considerations of the previous sections. This behaviour is most closely matched by an overlap function with $p=1.6$, against which we can compare the results. The simplest way to remove this excess activity is a retuning of the $p_{\perp 0}$ parameter of the MPI framework, in this case achieved by raising $p_{\perp 0}^{\mathrm{ref}}=2.085 \rightarrow 2.15 \mathrm{GeV}$. This rise does not greatly affect the relative slope of $a_{0}$, as constrained in Sec. 3.1. The results are shown in Fig. 11 for the same distributions as Fig. 10.

After this retuning, the log profile shows some promise. For the charged multiplicity 

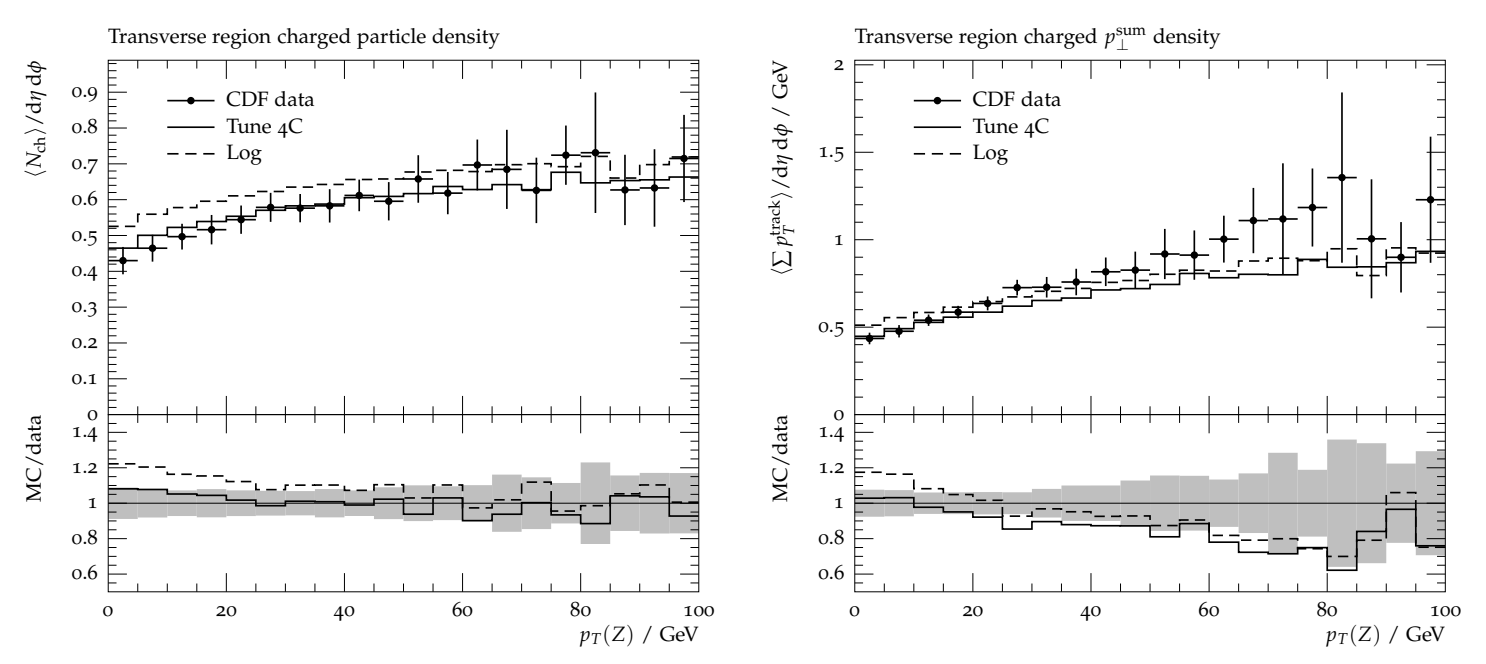

Figure 12: Charged number and sum- $p_{\perp}$ density in the transverse region of the underlying event in Drell-Yan

distribution, the tail now sits above the data with the overlap profile, while the match is improved with the log. This effect has already been seen in Fig. 88c, where the overlap and double Gaussian profiles "shoot up" in the tails, while the log profile gives a more gradual rise. The rise of the underlying event is almost exactly the same in the two different profiles. In the toward region, the rise is still too fast, and slightly worse than the unmodified tune. The log profile, here, does have slightly higher tails, consistent with a narrower matter profile in higher bins of $p_{\perp}^{\text {lead }}$, that do suggest a slightly better shape overall. The description in the transverse region is, in fact, improved, although a further decrease in activity, for example to improve agreement in the toward region, would likely push activity lower here, similar to the unmodified tune.

\subsection{Underlying event in Drell-Yan}

Studies can also be made on the underlying event in Drell-Yan processes. Here, a CDF study [60] is used, where a leading $\mathrm{Z}^{0}$ is reconstructed from the lepton pair. For simplicity, we stay with Tune $4 \mathrm{C}$ and the modified version of it, using the new matter profile, but noting that it has been shown to give too much activity at the Tevatron. Tune $2 \mathrm{C}$, also introduced in [9], is a tune based only on Tevatron data. It describes minimum-bias and jet-based underlying event studies well, but gives only limited agreement with the Drell-Yan results. In particular, the charged particle number and sum- $p_{\perp}$ densities in all regions are too low.

The results from PyтhiA are run through the Rivet analysis of this study [61]. The charged particle number and sum- $p_{\perp}$ densities in the transverse region, as a function of $p_{\perp}\left(\mathrm{Z}^{0}\right)$, are shown in Fig. 12. At first glance, it appears that Tune $4 \mathrm{C}$ does not do too badly, but, as above, it is known to give too much activity at the Tevatron. As expected from previous sections, the log profile, then, gives an increase in activity, which also occurs in the toward and away regions. It should be noted that the increase of the sum- $p_{\perp}$ density, relative to the charged number density, is dependent on other factors, such as colour reconnection, which differ between Tunes $4 \mathrm{C}$ and $2 \mathrm{C}$. Given this, the conclusions we can draw from this study are limited, but the $x$-dependent matter profile appears to be a step in the right 
direction.

\section{Summary and outlook}

There is both theoretical and experimental evidence suggesting that the wave function of high- $x$ partons should be narrower than that of low- $x$ ones. In this article, we have not tried to examine the underlying mechanism for this, but instead have modelled the effect using a simple Gaussian shape with a width that varies logarithmically with the $x$-value of the parton being probed. This is introduced as a new matter profile in the MPI framework of PYTHIA 8.

The framework, outlined in Sec. 2, is additionally formulated in terms of a physical size of the proton. Although introduced with a free parameter, $a_{1}$, regulating the importance of the logarithmic component, it can be fixed if it is assumed that the variation should account for the growth of the total cross section. For this to be the case, this parameter should lie in the region of $a_{1}=0.15$. In the studies made here, it has been considered a fixed quantity rather than a free parameter. The estimates of the proton size come out somewhat below current low-energy measurements, but as noted, the eikonalisation procedure neglects the diffractive components of the cross section.

The model gives a matter profile which in some ways is similar to the double Gaussian scenario. There is an increase in the matter at both small and large impact parameters, arising naturally due to the form of the eq. (9). The results are further changed, however, by the varying enhancement factors in the subsequent chain of MPI. An early tune to LHC data has been used to quantify the effects of this profile on minimum-bias and underlyingevent distributions. In particular, although the physical results are somewhat similar to an intermediate overlap profile, there are differences which give some indication that this profile could give rise to a viable tune to LHC data.

The case of MPI activity accompanying hard processes which do not contain final-state particles which can be created in MPI, such that the evolution covers the entire phase space, is interesting both in its own right, and as an illustration of the features of the model. The previous matter profiles give exactly the same impact parameter distribution, regardless of whether the underlying process is a $\mathrm{Z}^{0}$ or a $1 \mathrm{TeV}$ resonance. The new profile changes this situation. The $x$ dependence now leads to a situation where the higher-mass resonance will give rise to a narrower impact parameter profile, leading to changes in both the number of MPI and their $p_{\perp}$ spectrum. In comparisons to data, it leads to extra activity in the underlying event description of Drell-Yan processes, which appears to be a step in the right direction, in terms of describing Tevatron data.

The results, then, are promising. A more general tuning to data would help ascertain more clearly if this profile can improve the overall description, relative to the other profiles available. Future LHC studies on the underlying event in Drell-Yan processes would be a welcome addition, in order to further test the model. The framework will be released in the upcoming Pythia 8.150 version, along with the modified Tune 4C, where we hope it will be studied further by a wider community. 


\section{Acknowledgments}

This work was supported by the Marie Curie Early Stage Training program "HEP-EST" (contract number MEST-CT-2005-019626), the Marie Curie research training network "MCnet" (contract number MRTN-CT-2006-035606), and the Swedish Research Council (contract number 621-2010-3326).

\section{References}

[1] T. Sjöstrand and M. van Zijl, Phys. Rev. D36 (1987) 2019.

[2] H.-U. Bengtsson and T. Sjöstrand, Comput. Phys. Commun. 46 (1987) 43.

[3] T. Sjöstrand, S. Mrenna, and P. Skands, JHEP 05 (2006) 026, arXiv:hep-ph/0603175.

[4] D. Amati, A. Stanghellini, and S. Fubini, Nuovo Cim. 26 (1962) 896-954.

[5] V. A. Abramovskii, O. V. Kancheli, and V. N. Gribov, eConf C720906V1 (1972) 389-413.

[6] T. Sjöstrand and P. Z. Skands, JHEP 03 (2004) 053, arXiv:hep-ph/0402078.

[7] T. Sjöstrand and P. Z. Skands, Eur. Phys. J. C39 (2005) 129-154, arXiv:hep-ph/0408302.

[8] T. Sjöstrand, S. Mrenna, and P. Skands, Comput. Phys. Commun. 178 (2008) 852-867, arXiv:0710.3820 [hep-ph].

[9] R. Corke and T. Sjöstrand, arXiv:1011.1759 [hep-ph].

[10] B. Alessandro et al., arXiv:1101.1852 [hep-ex]

[11] A. Buckley et al., arXiv:1101.2599 [hep-ph].

[12] G. Marchesini and B. R. Webber, Phys. Rev. D38 (1988) 3419.

[13] G. Marchesini et al., Comput. Phys. Commun. 67 (1992) 465-508.

[14] J. M. Butterworth, J. R. Forshaw, and M. H. Seymour, Z. Phys. C72 (1996) 637-646, arXiv:hep-ph/9601371.

[15] I. Borozan and M. H. Seymour, JHEP 09 (2002) 015, arXiv:hep-ph/0207283.

[16] M. Bähr, S. Gieseke, and M. H. Seymour, JHEP 07 (2008) 076. arXiv:0803.3633 [hep-ph].

[17] M. Bähr et al., Eur. Phys. J. C58 (2008) 639-707, arXiv:0803.0883 [hep-ph].

[18] T. Gleisberg et al., JHEP 02 (2009) 007, arXiv:0811.4622 [hep-ph]. 
[19] M. G. Ryskin, A. D. Martin, and V. A. Khoze, Eur. Phys. J. C60 (2009) 249-264, arXiv:0812.2407 [hep-ph].

[20] A. Capella, U. Sukhatme, C.-I. Tan, and J. Tran Thanh Van, Phys. Rept. 236 (1994) 225-329.

[21] V. N. Gribov, Sov. Phys. JETP 26 (1968) 414-422.

[22] R. Engel, Z. Phys. C66 (1995) 203-214.

[23] R. Engel and J. Ranft, Phys. Rev. D54 (1996) 4244-4262, arXiv:hep-ph/9509373.

[24] F. W. Bopp, J. Ranft, R. Engel, and S. Roesler, Phys. Rev. C77 (2008) 014904, arXiv:hep-ph/0505035.

[25] K. Werner, F.-M. Liu, and T. Pierog, Phys. Rev. C74 (2006) 044902, arXiv:hep-ph/0506232.

[26] E.-J. Ahn, R. Engel, T. K. Gaisser, P. Lipari, and T. Stanev, Phys. Rev. D80 (2009) 094003, arXiv:0906.4113 [hep-ph].

[27] S. Ostapchenko, arXiv:1010.1869 [hep-ph].

[28] R. J. Glauber, "High-Energy Collision Theory." in Lectures in Theoretical Physics, W. E. Brittin and L. G. Dunham, eds., vol. I, pp. 315 - 414. Interscience, New York, 1959.

[29] E. Avsar, G. Gustafson, and L. Lönnblad, JHEP 07 (2005) 062, arXiv:hep-ph/0503181.

[30] E. Avsar, G. Gustafson, and L. Lönnblad, JHEP 01 (2007) 012, arXiv:hep-ph/0610157.

[31] E. Avsar, G. Gustafson, and L. Lönnblad, JHEP 12 (2007) 012, arXiv:0709.1368 [hep-ph].

[32] P. J. Mohr, B. N. Taylor, and D. B. Newell, Rev. Mod. Phys. 80 (2008) 633-730, arXiv:0801.0028 [physics.atom-ph].

[33] M. Froissart, Phys. Rev. 123 (1961) 1053-1057.

[34] A. Martin, Nuovo Cim. A42 (1965) 930-953.

[35] L. Lukaszuk and A. Martin, Nuovo Cim. A52 (1967) 122-145.

[36] A. Martin, Phys. Rev. D80 (2009) 065013, arXiv:0904.3724 [hep-ph].

[37] T. T. Wu, A. Martin, S. M. Roy, and V. Singh, arXiv:1011.1349 [hep-ph].

[38] T. Chou and C.-N. Yang, Phys.Rev. 170 (1968) 1591-1596.

[39] H. Cheng, J. Walker, and T. Wu, Phys.Rev. D9 (1974) 749-751. 
[40] R. Henzi and P. Valin, Phys. Lett. B132 (1983) 443-448.

[41] C. Bourrely, J. Soffer, and T. Wu, Nucl.Phys. B247 (1984) 15.

[42] L. Frankfurt, M. Strikman, and C. Weiss, Ann. Rev. Nucl. Part. Sci. 55 (2005) 403-465, arXiv: hep-ph/0507286.

[43] E. A. Kuraev, L. N. Lipatov, and V. S. Fadin, Sov. Phys. JETP 45 (1977) 199-204.

[44] I. I. Balitsky and L. N. Lipatov, Sov. J. Nucl. Phys. 28 (1978) 822-829.

[45] I. Balitsky, Nucl. Phys. B463 (1996) 99-160, arXiv:hep-ph/9509348.

[46] Y. Hatta, E. Iancu, L. McLerran, A. Stasto, and D. N. Triantafyllopoulos, Nucl. Phys. A764 (2006) 423-459, arXiv: hep-ph/0504182.

[47] A. H. Mueller, Nucl. Phys. B415 (1994) 373-385.

[48] A. H. Mueller and B. Patel, Nucl. Phys. B425 (1994) 471-488, arXiv:hep-ph/9403256.

[49] E. Avsar, JHEP 04 (2008) 033, arXiv:0803.0446 [hep-ph].

[50] C. Flensburg and G. Gustafson, JHEP 10 (2010) 014, arXiv:1004.5502 [hep-ph].

[51] E. Avsar, C. Flensburg, Y. Hatta, J.-Y. Ollitrault, and T. Ueda, arXiv:1009.5643 [hep-ph].

[52] M. Burkardt, Int. J. Mod. Phys. A18 (2003) 173-208, arXiv:hep-ph/0207047.

[53] M. Diehl, PoS DIS2010 (2010) 223, arXiv:1007.5477 [hep-ph].

[54] A. Donnachie and P. V. Landshoff, Phys. Lett. B296 (1992) 227-232, arXiv:hep-ph/9209205.

[55] L. Lönnblad, JHEP 05 (2002) 046, arXiv:hep-ph/0112284.

[56] G. A. Schuler and T. Sjöstrand, Phys. Rev. D49 (1994) 2257-2267.

[57] ATLAS Collaboration, G. Aad et al., Phys. Lett. B688 (2010) 21-42, arXiv: 1003.3124 [hep-ex].

[58] ATLAS Collaboration. ATLAS-CONF-2010-024.

[59] ATLAS Collaboration. ATLAS-CONF-2010-029 (2010).

[60] CDF Collaboration, D. Kar and R. Field. CDF Note 9351 (2008).

[61] A. Buckley et al., arXiv:1003.0694 [hep-ph]. 